\title{
Aminoácidos digestíveis verdadeiros de alguns alimentos protéicos determinados em galos cecotomizados e por equações de predição ${ }^{1}$
}

\section{Paulo Roberto Ost ${ }^{2}$, Paulo Borges Rodrigues ${ }^{3}$, Rilke Tadeu Fonseca de Freitas ${ }^{3}$, Elias Tadeu Fialho $^{3}$, Antônio Gilberto Bertechini ${ }^{3}$, Hunaldo Oliveira Silva ${ }^{4}$}

\author{
${ }^{1}$ Parte da tese de Doutorado do primeiro autor. Projeto financiado pelo CNPq \\ 2 Unicentro/PR. \\ ${ }^{3}$ Departamento de Zootecnia da UFLA. \\ ${ }^{4}$ EAFSC/SE
}

\begin{abstract}
RESUMO - Foram determinados os coeficientes de digestibilidade e os valores de aminoácidos digestíveis verdadeiros da soja integral tostada, soja integral micronizada e de cinco amostras de farelos de soja. Utilizou-se o método de alimentação forçada, com galos adultos cecotomizados. Foram utilizados 24 galos Leghorn adultos, com peso médio de $2348 \pm 165$ g. Cada alimento foi fornecido a seis galos, sendo o galo a unidade experimental, em duas repetições no tempo. Os animais foram forçados a ingerir $30 \mathrm{~g}$ do alimento-teste, seguidos de um período de 48 horas de coleta, após a alimentação forçada. Seis galos foram mantidos em jejum para determinação das perdas endógenas e metabólicas. Os valores de aminoácidos totais foram aproximadamente $10 \%$ inferiores àqueles apresentados pela literatura e os coeficientes de digestibilidade verdadeira dos aminoácidos essenciais e não-essenciais, de 91,3 e 90,6\%, respectivamente. Entre os aminoácidos essenciais dos farelos de soja, a isoleucina apresentou a menor digestibilidade média $(86,4 \%)$ e a fenilalanina, a maior digestibilidade $(95,5 \%)$. Quando estimados pelas equações de predição, observou-se que, de maneira geral, as equações propostas na literatura estrangeira não fizeram boas estimativas do conteúdo em aminoácidos de alimentos do grupo da soja. Com exceção da lisina e metionina, as equações propostas na literatura nacional fizeram boas estimativas, comparadas aos valores analisados.
\end{abstract}

Palavras-chave: alimentação forçada, digestibilidade, soja integral, soja micronizada, farelo de soja

\section{True digestible amino acids of some protein feedstuffs determined with cecectomized cockerels and by prediction equations}

\begin{abstract}
The true digestibility coefficients and the values of true digestible amino acids of whole toasted soybean, micronized soybean and five soybean meals samples were determined. The forced feeding method was used with cecectomized adult cockerels. A total of 24 adult Leghorn cockerels averaging $2348 \pm 165$ g were used. Each feedstuff was fed to six cockerels, with the cockerel the experimental unit, in two replicates in the time. A total of $30 \mathrm{~g}$ of the tested feedstuff was forced fed to cockerel, in a total of six replicates and the excreta collection was realized during $48 \mathrm{~h}$ after forced fed. Simultaneously six cockerels were maintained in fasting to determine the metabolic and endogenous losses. The values of total amino acids were about $10 \%$ lower than those presented by the literature and the true digestibility coefficients of the essential and non-essential amino acids of those feeds were 91.3 and 90.6\%, respectively. Among essential amino acids of soybean meals, isoleucine was the one with lower average digestibility (86.4\%) and phenylalanine, that which presented the higher digestibility (95.5\%). When estimated by the prediction equations, it was observed that, in general, the equations proposed in the international literature did not fit well the content of amino acids of the feedstuffs of the soybean group. However, except for the lysine and the methionine, the equations proposed in the national literature made good prediction in comparison to the analyzed values.
\end{abstract}

Key Words: forced feeding, digestibility, micronized soybean, soybean meal, whole toasted soybean

\section{Introdução}

Na formulação de rações para aves, as fontes protéicas correspondem a aproximadamente $25 \%$ do custo das rações, valor que pode ser alterado em função de possível desbalanço de aminoácidos. Neste contexto, o balanceamento deve ocorrer de modo que não ocorra falta ou excesso de qualquer aminoácido, visando ao seu completo aproveitamento. O desbalanço pode acarretar problemas na absorção e no aproveitamento dos aminoácidos, afetando o desempenho das aves e, conseqüentemente, causando prejuízos à exploração avícola. 
É notória a importância da soja e de seus subprodutos como matéria-prima na formulação de rações no Brasil, em razão de seu elevado valor nutritivo. Segundo Dale (1997), vários estudos têm sido realizados para se obter máximo aproveitamento das propriedades nutricionais da soja, posto que, na maioria das vezes, o farelo de soja é responsável por aproximadamente $70 \%$ da suplementação protéica nas rações.

As exigências protéicas são determinadas em função do atendimento das necessidades metabólicas em aminoácidos e nitrogênio. Quanto mais estreita for a relação entre o perfil de aminoácidos do alimento e o perfil corporal (animal), maior será o valor biológico do alimento e menor será a porcentagem de proteína necessária na ração. Esta relação depende da quantidade e digestibilidade dos aminoácidos presentes em determinado ingrediente.

Por outro lado, sabe-se bem que as quantidades de aminoácidos digestíveis nos alimentos são substancialmente menores que a quantidade total e, associado a isto, o desenvolvimento de ensaios de digestibilidade mais rápidos, bem como o aperfeiçoamento daqueles já existentes, tornou possível a condução de maior número de pesquisas sobre este assunto (Parsons, 1996).

Segundo Rostagno et al. (1995), o conceito de aminoácidos digestíveis é amplamente aceito para estimar a fração disponível de aminoácidos contidos nos alimentos. Embora grande variedade de métodos de ensaio de digestibilidade de aminoácidos possa ser usada, o mais usual é o ensaio de digestibilidade em galos adultos, referido como metodologia de Sibbald (1979), em que se utilizam galos cecotomizados. O uso de aves cecotomizadas na determinação dos coeficientes de digestibilidade dos aminoácidos caracteriza esta metodologia como a mais precisa, uma vez que permite eliminar a influência da flora microbiana presente nos cecos das aves e, assim, possíveis erros associados à absorção de aminoácidos no intestino grosso.

Várias pesquisas têm sido realizadas para determinar as variações no conteúdo de aminoácidos dos ingredientes, em função de sua composição proximal, especialmente da PB. O NRC (1998) apresenta uma série de equações lineares que podem ser utilizadas para se estimar o conteúdo de aminoácidos dos alimentos, em função de sua composição química, principalmente dos valores de PB. Portanto, objetivou-se neste trabalho determinar os coeficientes de digestibilidade dos aminoácidos e os aminoácidos digestíveis verdadeiros da soja e farelos e, a partir dos resultados experimentais obtidos, validar equações de predição que foram desenvolvidas por outros autores.

\section{Material e Métodos}

Para determinar os coeficientes de digestibilidade dos aminoácidos e os aminoácidos digestíveis verdadeiros da soja e subprodutos, foi conduzido um ensaio biológico na sala de metabolismo do Setor de Avicultura do Departamento de Zootecnia da Universidade Federal de Lavras, em Lavras, MG. A temperatura interna da sala de metabolismo foi mantida em $23 \pm 2^{\circ} \mathrm{C}$.

Foi adaptado o método de alimentação forçada proposto por Sibbald (1979), utilizando-se 24 galos Leghorn adultos cecotomizados, com 30 meses de idade e peso médio de $2.348 \pm 165 \mathrm{~g}$. Os animais foram forçados a ingerir sete alimentos protéicos à base de soja, adquiridos em empresas comerciais, sendo cinco marcas de farelo de soja encontradas no comércio (denominados farelos de soja 1, 2, 3, 4 e 5), soja integral tostada e soja integral micronizada.

O procedimento cirúrgico de cecectomia foi efetuado no Hospital Veterinário da UFLA, por meio de laparotomia abdominal e anestesia local, conforme metodologia descrita por Pupa et al. (1998). Três meses após a cirurgia, os galos foram submetidos à fase de adaptação, antes de se iniciar o período experimental.

Cada um dos sete alimentos foi fornecido a três galos, em duas repetições no tempo, perfazendo seis repetições por tratamento. Cada galo foi alojado individualmente e constituiu uma unidade experimental. Adotou-se um procedimento para que cada ave não recebesse o mesmo alimento duas vezes. Simultaneamente, em cada uma das repetições no tempo, foram mantidos três galos em jejum, para determinação das perdas endógenas e metabólicas. Adotou-se intervalo de sete dias entre as repetições no tempo, para descanso dos galos e recuperação de seu peso, momento em que os animais receberam uma ração de terminação de frangos de corte, ou seja, de alta densidade nutricional, para frangos de corte.

Antes do período experimental, os galos foram alojados em gaiolas individuais e submetidos a um período de adaptação, no qual receberam alimentação em dois turnos de 1 hora (8 e 16h), para que se promovesse a dilatação do papo - processo importante para diminuir a ocorrência de regurgitações após a alimentação forçada, durante o período experimental, pois, quando o galo regurgite, há perda da repetição.

Posteriormente, as aves foram mantidas em jejum por 24 horas, com o objetivo de esvaziar o trato digestivo e, então, forçadas a ingerir $30 \mathrm{~g}$ do alimento-teste, por meio de um funil-sonda introduzido via esôfago até o papo, fornecida aos galos às $8 \mathrm{~h}$. Nesse momento, foram colocadas as 
bandejas coletoras de excretas sob as gaiolas, devidamente revestidas com plásticos, a fim de evitar perdas e facilitar a coleta. A coleta total das excretas foi realizada duas vezes ao dia (8 e 16h), para evitar fermentações, por um período de 48 horas após o início do fornecimento dos alimentos, totalizando quatro coletas.

As excretas coletadas foram acondicionadas em frascos de vidro com tampas, devidamente pesados e identificados, e, então, mantidas em congelador $\left(-10^{\circ} \mathrm{C}\right)$ até o final do período de coleta. Estas amostras foram homogeneizadas e levadas ao laboratório para análises de MS e nitrogênio, por meio das metodologias descritas por Silva (1990), após pré-secagem em estufa ventilada a $55^{\circ} \mathrm{C}$, por 72 horas ou até peso constante. O conteúdo de aminoácidos foi determinado pelo método de hidrólise ácida, com utilização da cromatografia líquida de alta performance (HPLC), com exceção do triptofano, que não resiste à hidrólise ácida. As análises foram realizadas no Laboratório de Pesquisa Animal do DZO/UFLA, com exceção do amido, que foi analisado no Departamento de Ciências dos Alimentos.

Uma vez conhecida a quantidade de aminoácidos ingeridos e excretados, bem como a fração endógena determinada com os galos em jejum, determinaram-se os coeficientes de digestibilidade verdadeira de cada aminoácido (CDVaa), por meio da fórmula proposta por Rostagno \& Featherston (1977):

$$
\begin{gathered}
\text { CDVaa }(\%)=\text { aa ingerido }- \text { aa excretado }+ \text { aa da } \\
\frac{\text { fração endógena }}{\text { aa ingerido (em gramas) }} \times 100
\end{gathered}
$$

Posteriormente, foi calculado o conteúdo de aminoácidos digestíveis verdadeiros de cada alimento.

As equações propostas para estimar o conteúdo em aminoácidos totais da soja integral tostada, da soja micronizada e dos farelos de soja, propostas por Rodrigues et al. (2002), são:

$$
\begin{aligned}
& \text { Lisina }=-0,32161+0,06731 \mathrm{~PB}\left(\mathrm{R}^{2}=0,99\right) ; \\
& \text { Metionina = 0,02263 +0,01323PB }\left(\mathrm{R}^{2}=0,96\right) ; \\
& \text { Metionina + cistina }=0,07894+0,02563 \mathrm{~PB}\left(\mathrm{R}^{2}=0,94\right) ; \\
& \text { Treonina }=0,20930+0,03533 \mathrm{~PB}\left(\mathrm{R}^{2}=0,95\right) ; \\
& \text { Arginina }=-2,19880+0,11509 \mathrm{~PB}+0,01702 \mathrm{EE}\left(\mathrm{R}^{2}=0,99\right) ; \\
& \text { Arginina }^{2}=-0,39589+0,08022 \mathrm{~PB}\left(\mathrm{R}^{2}=0,98\right),
\end{aligned}
$$

Para o conteúdo em aminoácidos digestíveis desses alimentos, as equações propostas por esses autores são:

Lisina $=-0,29472+0,06319 P B\left(R^{2}=0,98\right)$;

Metionina $=0,02884+0,01228 \mathrm{~PB}\left(\mathrm{R}^{2}=0,93\right)$;
Metionina + cistina $=0,08974+0,02220 \mathrm{~PB}\left(\mathrm{R}^{2}=0,91\right)$

Treonina $=0,15813+0,03235 \mathrm{~PB}\left(\mathrm{R}^{2}=0,95\right)$;

Arginina $=-2,7699+0,12416 \mathrm{~PB}+0,02312 \mathrm{EE}\left(\mathrm{R}^{2}=0,99\right)$;

Arginina $^{2}=-0,32129+0,07681 \mathrm{~PB}\left(\mathrm{R}^{2}=0,96\right)$.

O NRC (1998) descreve equações de predição do conteúdo em aminoácidos totais, específicas para o farelo de soja, conforme se apresenta:

$$
\begin{aligned}
& \text { Lisina }=-0,0810+0,0644 \text { PB }(r=0,78) \\
& \text { Metionina }=0,0170+0,0141 \text { PB }(r=0,65) ; \\
& \text { Metionina + cistina }=0,1470+0,0263 \text { PB }(r=0,57)
\end{aligned}
$$$$
\text { Treonina }=0,0810+0,0381 \mathrm{~PB}(\mathrm{r}=0,81) \text {, em que r é o }
$$
coeficiente de correlação entre as variáveis (NRC, 1998).

Para verificar a aplicabilidade dessas equações, os valores estimados para aminoácidos totais e digestíveis foram comparados aos analisados (aminoácidos totais) e aos calculados a partir dos coeficientes de digestibilidade obtidos nos ensaios de metabolismo (aminoácidos digestíveis). Posteriormente, verificou-se a diferença percentual relativa entre os valores estimados e os encontrados neste trabalho e, ainda, se os valores estimados se localizaram dentro de uma amplitude considerada aceitável pela literatura, para cada aminoácido.

\section{Resultados e Discussão}

A composição em aminoácidos essenciais e não-essenciais (Tabelas 1 e 2, respectivamente) das amostras dos farelos de soja 1, 2, 3, 4 e 5 e das sojas integrais (tostada e micronizada) comprova variação de alguns aminoácidos (valores superior, inferior ou semelhante aos descritos na literatura). O teor médio de lisina dos farelos de soja, por exemplo, foi 13,31; 11,07; 10,41 e 10,74\% inferior aos apresentados por Rostagno et al. (2000), Rodrigues et al. (2002), NRC (1994) e Brasil (2000), respectivamente.

A média da lisina das amostras de soja integral (tostada e micronizada) diferiu da descrita por Rhône Poulenc (1993), NRC (1994) e Fischer Jr. et al. (1998), sendo 11,88 e $14,18 \%$ superior à relatada por Rodrigues et al. (2002) e Rostagno et al. (2000), respectivamente, porém semelhante à descrita em Brasil (2000). Os valores da soja micronizada obtidas neste trabalho foram semelhantes àqueles descritos por Rodrigues et al. (2002) e Rostagno et al. (2000), observando-se a maior diferença para a soja tostada.

Nota-se que não só os valores de lisina, mas também dos demais aminoácidos essenciais, variaram em composição, dentro de amostras, como observado para o farelo de soja neste trabalho, o que também é citado por 
Rostagno et al. (1995). Estas observações reforçam as inferências de Albino et al. (1987) de que a composição nutricional dos alimentos é influenciada pela variação nos solos e climas em que são cultivados, pelas variedades e pelo processamento a que são submetidos.

De modo geral, verifica-se que os valores de aminoácidos essenciais e não-essenciais totais dos alimentos estudados foram menores (aproximadamente 10\%) que os reportados na maioria das referências consultadas (Rhône Poulenc, 1993; NRC, 1994; Fischer Jr. et al., 1998; Rodrigues et al., 2002; Rostagno et al., 2000; Brasil, 2000), resultado provavelmente atribuído à possível influência das variedades de soja recentemente utilizadas ou ao próprio processamento destes ingredientes.

Nas cinco marcas comerciais de farelo de soja estudadas, os aminoácidos essenciais treonina, arginina, histidina, isoleucina e valina apresentaram coeficientes médios de digestibilidade verdadeira inferiores a 90\% (Tabela 3). Entre os aminoácidos não-essenciais, apenas a alanina e o ácido aspártico tiveram digestibilidade verdadeira menor que $90 \%$ (Tabela 4).

Entre os aminoácidos essenciais dos farelos de soja, a isoleucina foi a que teve menor digestibilidade média $(86,4 \%)$ e, entre os não-essenciais, a alanina apresentou o menor coeficiente de digestibilidade médio (86,2\%). A fenilalanina foi o aminoácido essencial que obteve o maior coeficiente de digestibilidade médio (95,5\%) e a cistina, o não-essencial com maior digestibilidade (94,6\%). Os resultados obtidos por Rostagno et al. (1995), Fischer Jr. et al. (1998) e Rodrigues et al. (2002) não foram tão altos quanto aos obtidos neste trabalho.

Ao serem contrastadas as digestibilidades médias dos aminoácidos essenciais e não-essenciais, nota-se superioridade dos aminoácidos essenciais sobre os não-

Tabela 1 - Conteúdo de aminoácidos essenciais totais da soja integral e subprodutos (\%MN)

Table 1 - Total indispensable amino acids content of the full fat soybean and byproducts (\% as-fed basis)

\begin{tabular}{|c|c|c|c|c|c|c|c|c|c|c|c|c|c|}
\hline \multirow[t]{2}{*}{$\begin{array}{l}\text { Alimento } \\
\text { Feed }\end{array}$} & \multicolumn{13}{|c|}{$\begin{array}{l}\text { Aminoácido } \\
\text { Amino acid }\end{array}$} \\
\hline & $\begin{array}{l}\text { MS } \\
D M\end{array}$ & $\begin{array}{l}\mathrm{PB} \\
C P\end{array}$ & Lys & Met & Met + Cys & Thr & Arg & Hys & Ile & Leu & Phe & Val & $\begin{array}{l}\text { TAAE } \\
\text { TIAA }\end{array}$ \\
\hline $\begin{array}{l}\text { Farelo de soja } 1 \\
\text { Soybean meal } 1\end{array}$ & 89,34 & 46,44 & 2,29 & 0,48 & 1,34 & 1,79 & 3,16 & 1,23 & 1,89 & 3,05 & 1,87 & 1,91 & 19,99 \\
\hline $\begin{array}{l}\text { Farelo de soja } 2 \\
\text { Soybean meal } 2\end{array}$ & 90,08 & 47,38 & 2,60 & 0,50 & 1,12 & 1,70 & 3,51 & 1,31 & 1,86 & 3,03 & 1,88 & 1,89 & 19,39 \\
\hline $\begin{array}{l}\text { Farelo de soja } 4 \\
\text { Soybean meal } 4\end{array}$ & 89,64 & 48,21 & 2,40 & 0,51 & 1,20 & 1,80 & 3,50 & 1,56 & 1,86 & 3,07 & 1,89 & 1,87 & 19,66 \\
\hline $\begin{array}{l}\text { Farelo de soja } 5 \\
\text { Soybean meal } 5\end{array}$ & 90,03 & 47,57 & 2,34 & 0,57 & 1,28 & 1,69 & 3,12 & 1,24 & 1,86 & 3,15 & 1,86 & 1,89 & 19,00 \\
\hline $\begin{array}{l}\text { Soja integral tostada } \\
\text { Toasted full fat soybean }\end{array}$ & 92,72 & 34,39 & 2,92 & 0,41 & 1,20 & 1,54 & 3,38 & 2,25 & 1,58 & 3,01 & 1,91 & 1,96 & 20,17 \\
\hline
\end{tabular}

TAAE - Total dos aminoácidos essenciais (TIAA - Total indispensable amino acids).

Tabela 2 - Conteúdo de aminoácidos não-essenciais totais da soja integral e subprodutos (\%MN)

Table 2 - Total dispensable amino acids content of the fullfat soybean and byproducts (\% as-fed basis)

\begin{tabular}{|c|c|c|c|c|c|c|c|c|}
\hline \multirow[b]{2}{*}{$\begin{array}{l}\text { Alimento } \\
\text { Feed }\end{array}$} & \multicolumn{8}{|c|}{$\begin{array}{l}\text { Aminoácido } \\
\text { Amino acid }\end{array}$} \\
\hline & Cys & Ala & Asp & Glu & Glu & Ser & Tyr & $\begin{array}{c}\text { TAANE } \\
\text { TDAA }\end{array}$ \\
\hline Farelo de soja 1 (Soybean meal 1) & 0,86 & 2,14 & 4,24 & 6,36 & 1,73 & 2,05 & 1,74 & 20,33 \\
\hline Farelo de soja 2 (Soybean meal 2) & 0,62 & 2,07 & 4,39 & 6,52 & 1,89 & 2,02 & 1,47 & 18,98 \\
\hline Farelo de soja 3 (Soybean meal 3) & 0,72 & 2,06 & 4,17 & 6,37 & 1,89 & 1,99 & 1,98 & 19,20 \\
\hline Soja integral tostada (Toasted full fat soybean) & 0,79 & 2,03 & 4,46 & 5,40 & 1,61 & 2,18 & 1,88 & 18,35 \\
\hline Soja micronizada (Micronized full fat soybean) & 0,47 & 1,53 & 3,33 & 7,36 & 2,18 & 1,64 & 1,57 & 18,09 \\
\hline
\end{tabular}

TAANE - Total dos aminoácidos não essenciais (TDAA - Total dispensable amino acids). 
Tabela 3 - Coeficientes de digestibilidade verdadeira dos aminoácidos essenciais da soja integral e subprodutos, determinados com galos cecotomizados

Table 3 - $\quad$ True digestibility coefficients of the indispensable amino acids of the full fat soybean and byproducts determined with cecectomized cockerels

\begin{tabular}{|c|c|c|c|c|c|c|c|c|c|c|c|}
\hline \multirow[t]{2}{*}{$\begin{array}{l}\text { Alimento } \\
\text { Feed }\end{array}$} & \multicolumn{11}{|c|}{$\begin{array}{c}\text { Coeficiente de digestibilidade verdadeira }(\%)^{1} \\
\text { True digestibility coefficient }\end{array}$} \\
\hline & $\begin{array}{l}\text { Lys } \\
(\%)\end{array}$ & $\begin{array}{l}\text { Met } \\
(\%)\end{array}$ & $\begin{array}{c}\text { Met }+ \text { Cys } \\
(\%)\end{array}$ & $\begin{array}{l}\text { Thr } \\
(\%)\end{array}$ & $\begin{array}{l}\text { Arg } \\
(\%)\end{array}$ & $\begin{array}{l}\text { Hys } \\
(\%)\end{array}$ & $\begin{array}{l}\text { Ile } \\
\text { (\%) }\end{array}$ & $\begin{array}{l}\text { Leu } \\
\text { (\%) }\end{array}$ & $\begin{array}{l}\text { Phe } \\
(\%)\end{array}$ & $\begin{array}{l}\text { Val } \\
(\%)\end{array}$ & $\begin{array}{c}\text { TAAE } \\
\text { TIAA } \\
(\%)\end{array}$ \\
\hline Farelo de soja 1 (Soybean meal 1) & 95,9 & 97,0 & 96,1 & 89,9 & 85,7 & 86,9 & 86,9 & 91,1 & 93,8 & 92,6 & 91,6 \\
\hline Farelo de soja 2 (Soybean meal 2) & 90,2 & 94,3 & 93,3 & 85,2 & 89,1 & 89,9 & 87,3 & 91,9 & 94,7 & 84,8 & 90,1 \\
\hline Farelo de soja 3 (Soybean meal 3) & 93,0 & 90,1 & 92,3 & 88,1 & 89,5 & 89,6 & 86,4 & 92,0 & 95,5 & 87,5 & 90,4 \\
\hline Farelo de soja 4 (Soybean meal 4) & 94,2 & 95,7 & 97,1 & 87,4 & 92,6 & 88,5 & 84,6 & 91,2 & 97,0 & 82,4 & 91,1 \\
\hline Farelo de soja 5 (Soybean meal 5) & 91,7 & 96,9 & 94,7 & 89,7 & 90,7 & 92,9 & 86,9 & 93,7 & 96,4 & 90,2 & 92,4 \\
\hline Soja integral tostada (Toasted integral soybean) & 90,5 & 96,5 & 97,0 & 90,1 & 87,1 & 86,1 & 89,2 & 89,3 & 90,1 & 90,1 & 90,8 \\
\hline Soja micronizada (Micronized soybean) & 88,6 & 94,7 & 96,8 & 87,3 & 90,0 & 91,4 & 91,0 & 93,4 & 97,9 & 87,7 & 91,9 \\
\hline
\end{tabular}

TAAE - Total dos aminoácidos essenciais (TIAA - Total indispensable amino acids).

Tabela 4 - Coeficientes de digestibilidade verdadeira dos aminoácidos não essenciais da soja integral e subprodutos, determinados com galos adultos cecotomizados

Table 4 - True digestibility coefficients of the dispensable amino acids of the full fat soybean and byproducts, determined with cecectomized cockerels

\begin{tabular}{|c|c|c|c|c|c|c|c|}
\hline \multirow[t]{2}{*}{$\begin{array}{l}\text { Alimento } \\
\text { Feed }\end{array}$} & \multicolumn{7}{|c|}{$\begin{array}{c}\text { Coeficiente de digestibilidade verdadeira (\%) } \\
\text { True digestibility coefficient (\%) }\end{array}$} \\
\hline & $\begin{array}{l}\text { Cys } \\
(\%)\end{array}$ & $\begin{array}{l}\text { Ala } \\
(\%)\end{array}$ & $\begin{array}{l}\text { Asp } \\
(\%)\end{array}$ & $\begin{array}{l}\text { Glu } \\
\text { (\%) }\end{array}$ & $\begin{array}{l}\text { Ser } \\
(\%)\end{array}$ & $\begin{array}{l}\text { Tyr } \\
(\%)\end{array}$ & $\begin{array}{c}\text { TAANE } \\
\text { TDAA } \\
(\%)\end{array}$ \\
\hline Farelo de soja 1 (Soybean meal 1) & 95,1 & 81,0 & 89,1 & 91,7 & 91,8 & 91,7 & 89,1 \\
\hline Farelo de soja 2 (Soybean meal 2) & 92,3 & 88,0 & 89,7 & 90,6 & 87,9 & 90,1 & 89,9 \\
\hline Farelo de soja 3 (Soybean meal 3) & 94,6 & 86,2 & 89,1 & 91,1 & 90,1 & 89,4 & 89,9 \\
\hline Farelo de soja 4 (Soybean meal 4) & 98,5 & 85,9 & 88,5 & 90,2 & 90,6 & 86,1 & 90,1 \\
\hline Farelo de soja 5 (Soybean meal 5) & 92,5 & 90,0 & 89,2 & 91,8 & 90,1 & 89,7 & 90,7 \\
\hline Soja int. tostada (Toasted integral soybean) & 97,4 & 92,8 & 95,8 & 90,7 & 91,7 & 89,9 & 92,6 \\
\hline Soja micronizada (Micronized soybean) & 98,9 & 82,7 & 90,5 & 95,2 & 89,9 & 95,0 & 92,3 \\
\hline
\end{tabular}

TAANE - Total dos aminoácidos não essenciais (TDAA - Total dispensable amino acids).

essenciais, o que pode indicar um mecanismo do organismo das aves para o melhor aproveitamento dos aminoácidos nutricionalmente indispensáveis.

Quando se comparam os coeficientes de digestibilidade dos farelos de soja obtidos neste trabalho e na literatura, constata-se variabilidade na maioria dos aminoácidos avaliados (Albino et al., 1992; Degussa, 1993; Rhône Poulenc, 1993; Fischer Jr. et al., 1998; Rostagno et al, 2000; Rodrigues et al., 2002), semelhantemente ao observado para a composição em aminoácidos totais, indicando a possível influência do processamento dos farelos sobre estes valores.

As variações reportadas pela literatura e mesmo entre os alimentos avaliados nesta pesquisa estão, certamente, associadas ao processamento de calor a que estes alimentos são submetidos. O excesso de calor durante o processamento pode reduzir a disponibilidade dos aminóacidos por mudanças fisico-químicas, como derivação da lisina, oxidação do enxofre na cisteína e metionina e ligações cruzadas em amidas e carboxilas (Araba \& Dale, 1990). Por outro lado, deve-se destacar que, também na composição em aminoácidos totais, foram observadas diferenças em relação à literatura consultada, possivelmente em razão de variações relacionadas às amostras provenientes de diferentes locais e processamentos, particularmente as de farelo de soja, aos sistemas de cultivo, à adubação, ao clima, entre outros.

O coeficiente médio de digestibilidade verdadeira da lisina dos farelos de soja neste ensaio foi semelhante ao reportado por Rodrigues et al. (2002) e, em média, 2,15; 5,4 e $1,47 \%$ superior àqueles apresentados por Rostagno et al. (2000), NRC (1994) e Bellaver et al. (1998), respectivamente. Estas variações podem decorrer, no entanto, dos diferentes procedimentos experimentais, como quantidade do alimento ingerido e pureza do material coletado, que está associado ao número de coletas por dia, e de possíveis diferenças em análises laboratoriais. 
Evidencia-se, a partir das variações observadas nos coeficientes de digestibilidade dos aminoácidos entre os alimentos, a grande importância da digestibilidade desses nutrientes, quando da formulação de rações, para que se possa obter máximo desempenho das aves. Este fator é intensificado quando se trabalha com alimentos alternativos ao milho e o farelo de soja - as sojas integrais, por exemplo.

Com base nos coeficientes de digestibilidade determinados para cada aminoácido no ensaio biológico, calculou-se o conteúdo de aminoácidos digestíveis dos alimentos, apresentados nas Tabelas 5 (essenciais) e 6 (nãoessenciais). Estes valores são de grande utilidade, pois, na formulação de rações para aves, o uso da composição em aminoácidos digestíveis já é amplamente empregado.
De acordo com Degussa (1993), o valor de uma proteína alimentar depende de seu conteúdo em aminoácidos essenciais e sua disponibilidade para o metabolismo das aves. Quando se consideram os aminoácidos em função de sua digestibilidade, pode-se fornecê-los adequadamente aos animais. A formulação de rações à base de aminoácidos digestíveis com ingredientes alternativos proporciona melhores desempenhos que as formuladas à base de aminoácidos totais (Tankslei \& Knabe, 1984; Parsons et al., 1995).

Assim como observado para o conteúdo total de aminoácidos dos alimentos, houve variabilidade entre os valores de aminoácidos digestíveis determinados neste trabalho e aqueles relatados por NRC (1994), Fischer Jr. et al. (1998), Brasil (2000), Rodrigues et al. (2002) e Rostagno

Tabela 5 - Conteúdo de aminoácidos essenciais digestíveis da soja integral e subprodutos (\% MN)

Table 5 - Digestible indispensable amino acids content of the full fat soybean and byproducts (\% as-fed basis)

\begin{tabular}{|c|c|c|c|c|c|c|c|c|c|c|c|c|c|}
\hline \multirow[t]{2}{*}{$\begin{array}{l}\text { Alimento } \\
\text { Feed }\end{array}$} & \multicolumn{13}{|c|}{$\begin{array}{c}\text { Aminoácido }{ }^{1,2} \\
\text { Amino acid }\end{array}$} \\
\hline & $\begin{array}{l}\text { MS } \\
D M\end{array}$ & $\begin{array}{l}\mathrm{PB} \\
\mathrm{CP}\end{array}$ & Lys & Met & Met + Cys & Thr & Arg & Hys & Ile & Leu & Phe & Val & $\begin{array}{l}\text { TAAE } \\
\text { TIAA }\end{array}$ \\
\hline $\begin{array}{l}\text { Farelo de soja } 1 \\
\text { Soybean meal } 1\end{array}$ & 89,3 & 46,4 & 2,20 & 0,47 & 1,29 & 1,61 & 2,71 & 1,07 & 1,64 & 2,78 & 1,75 & 1,77 & 18,31 \\
\hline $\begin{array}{l}\text { Farelo de soja } 2 \\
\text { Soybean meal } 2\end{array}$ & 90,0 & 47,3 & 2,35 & 0,47 & 1,04 & 1,45 & 3,13 & 1,18 & 1,62 & 2,78 & 1,78 & 1,60 & 17,47 \\
\hline $\begin{array}{l}\text { Farelo de soja } 4 \\
\text { Soybean meal } 4\end{array}$ & 89,6 & 48,2 & 2,26 & 0,49 & 1,17 & 1,57 & 3,24 & 1,38 & 1,57 & 2,80 & 1,83 & 1,54 & 17,91 \\
\hline $\begin{array}{l}\text { Farelo de soja } 5 \\
\text { Soybean meal } 5\end{array}$ & 90,0 & 47,5 & 2,15 & 0,55 & 1,21 & 1,52 & 2,83 & 1,15 & 1,62 & 2,95 & 1,79 & 1,70 & 17,56 \\
\hline $\begin{array}{l}\text { Soja integral tostada } \\
\text { Toasted full fat soybean }\end{array}$ & 92,7 & 34,3 & 2,64 & 0,40 & 1,16 & 1,39 & 2,94 & 1,94 & 1,41 & 2,69 & 1,72 & 1,77 & 14,66 \\
\hline
\end{tabular}

TAAE - Total dos aminoácidos essenciais (TIAA - Total indispensable amino acids).

1 Valores calculados pelos coeficientes de digestibilidade determinados in vivo e conteúdo de aminoácidos totais dos alimentos.

1 Values calculated by the digestibility coefficients determined "in vivo" and total amino acids content of the feeds.

Tabela 6 - Conteúdo de aminoácidos não essenciais digestíveis da soja e subprodutos (\%MN)

Table 6 - Digestible dispensable amino acids content of the full fat soybean and byproducts (\% as-fed basis)

\begin{tabular}{|c|c|c|c|c|c|c|c|}
\hline \multirow[t]{2}{*}{$\begin{array}{l}\text { Alimento } \\
\text { Feed }\end{array}$} & \multicolumn{7}{|c|}{$\begin{array}{c}\text { Aminoácido }{ }^{1,2} \\
\text { Amino acid }\end{array}$} \\
\hline & Cys & Ala & Asp & Glu & Ser & Ty r & $\begin{array}{c}\text { TAANE } \\
\text { TDAA }\end{array}$ \\
\hline Farelo de soja 1 (Soybean meal 1) & 0,82 & 1,73 & 3,78 & 5,83 & 1,88 & 1,60 & 18,11 \\
\hline Farelo de soja 2 (Soybean meal 2) & 0,57 & 1,82 & 3,94 & 5,91 & 1,78 & 1,32 & 17,06 \\
\hline Farelo de soja 3 (Soybean meal 3) & 0,68 & 1,78 & 3,72 & 5,80 & 1,79 & 1,77 & 17,26 \\
\hline Farelo de soja 4 (Soybean meal 4) & 0,68 & 1,71 & 3,79 & 5,00 & 1,77 & 1,58 & 16,46 \\
\hline Farelo de soja 5 (Soybean meal 5) & 0,66 & 1,85 & 3,37 & 6,48 & 1,75 & 1,51 & 17,41 \\
\hline Soja integral tostada (Toasted full fat soybean) & 0,77 & 1,88 & 4,27 & 4,90 & 2,00 & 1,69 & 16,99 \\
\hline Soja micronizada (Micronized soybean) & 0,46 & 1,27 & 3,01 & 7,01 & 1,47 & 1,49 & 16,70 \\
\hline
\end{tabular}

TAANE - Total dos aminoácidos não essenciais (TDAA - Total dispensable amino acids).

${ }^{1}$ Valores calculados pelos coeficientes de digestibilidade determinados in vivo e conteúdo de aminoácidos totais dos alimentos.

1 Values calculated by the digestibility coefficients determined "in vivo" and total amino acids content of the feedstuffs. 
et al. (2000), para o farelo de soja, e entre os reportados nesta pesquisa e aqueles reportados por Fischer Jr. et al. (1998), Rodrigues et al. (2002) e Rostagno et al. (2000), para as sojas integrais, tanto para aminoácidos essenciais como nãoessenciais. Estas diferenças podem estar associadas à variação observada tanto no conteúdo total quanto nos coeficientes de digestibilidade determinados, o que pode, conseqüentemente, resultar em variáveis conteúdos de aminoácidos digestíveis.

Observa-se que, apesar de o teor médio de lisina total ser estimado em $10,7 \%$ superior ao valor analisado, as estimativas individuais dos aminoácidos em cada alimento foram, em sua maioria, superiores a $15,0 \%$ quando comparadas aos valores analisados, com exceção do farelo de soja 2 e da soja micronizada (11,4 e 12,4\% superiores, respectivamente) e da soja integral tostada (30,8\% inferior) (Tabela 7). Analisando a média da lisina total somente dos farelos de soja, nota-se que a estimativa feita pela equação de predição não foi adequada, uma vez que, neste trabalho, a equação superestimou o conteúdo de lisina dos farelos em 19,7\%.

No entanto, quando se compara o valor médio de lisina dos farelos de soja, estimado pela equação (3,22\%), com o de lisina total (3,03 e 3,15\%), apresentado por Rodrigues et al (2002) e Rostagno et al. (2000), verifica-se que a principal diferença decorre, possivelmente, da análise química dos aminoácidos, evidenciando a aplicabilidade das equações avaliadas. É necessário, porém, mais estudos para se recomendar uma aplicação segura destas equações.

A estimativa média da metionina total foi $21,8 \%$ superior, comprovando que, tanto em média quanto individualmente, o uso das equações na estimativa do conteúdo deste aminoácido nos alimentos avaliados nesta pesquisa não foi adequado. Por outro lado, quando se considera a metionina + cistina, a estimativa conjunta deste aminoácido foi mais próxima ao valor médio analisado (1,5\%), observando-se o mesmo para a treonina (2,1\%), quando se avaliou apenas a média dos alimentos. No entanto, quando se consideram as sojas integrais (tostada e micronizada) individualmente, nota-se que, para a metionina + cistina, as estimativas não foram muito precisas, sendo aproximadamente $20 \%$ inferior ou superior aos valores analisados, respectivamente. A treonina apresentou valores estimados adequados, com estimativas de até aproximadadmente $10 \%$ superiores ou inferiores aos valores analisados.

A estimativa média de arginina, pelas duas equações propostas, foi 1,4 e 3,4\%, respectivamente, superior ao valor médio analisado (Tabela 7). Entretanto, com base no valor médio analisado das cinco amostras de farelo de soja, observou-se que as estimativas para metionina + cistina, treonina e arginina foram, em média, 3,8; 3,5; 3,1 e 5,2\% superiores aos valores analisados, respectivamente, para

Tabela 7 - Estimativas do conteúdo de LYS, MET, MET + CYS, THR e ARG totais da soja integral e subprodutos, por meio de equações de predição em função da composição química dos alimentos $(\% \mathrm{MS})^{1}$

Table 7 - Estimates of total LYS, MET, MET + CYS, THR and ARG contents of the full fat soybean and byproducts by the prediction equations based on chemical composition (\% DM)

\begin{tabular}{|c|c|c|c|c|c|c|c|c|c|c|c|c|}
\hline \multirow{2}{*}{$\begin{array}{l}\text { Alimento } \\
\text { Feed }\end{array}$} & \multicolumn{2}{|c|}{ Lys } & \multicolumn{2}{|c|}{ Met } & \multicolumn{2}{|c|}{ Met + Cys } & \multicolumn{2}{|c|}{ Thr } & \multicolumn{2}{|c|}{ Arg } & \multicolumn{2}{|c|}{$\operatorname{Arg}^{2}$} \\
\hline & Obs. $^{2}$ & $\mathrm{Est}^{3}$. & Obs. & Est. & Obs. & Est. & Obs. & Est. & Obs. & Est. & Obs. & Est. \\
\hline $\begin{array}{l}\text { Farelo de soja } 1 \\
\text { Soybean meal } 1\end{array}$ & 2,56 & 3,18 & 0,54 & 0,71 & 1,50 & 1,41 & 2,00 & 2,05 & 3,54 & 3,77 & 3,54 & 3,83 \\
\hline $\begin{array}{l}\text { Farelo de soja } 2 \\
\text { Soybean meal } 2\end{array}$ & 2,89 & 3,22 & 0,56 & 0,72 & 1,24 & 1,43 & 1,89 & 2,07 & 3,90 & 3,82 & 3,90 & 3,88 \\
\hline $\begin{array}{l}\text { Farelo de soja } 3 \\
\text { Soybean meal } 3\end{array}$ & 2,70 & 3,17 & 0,57 & 0,71 & 1,39 & 1,41 & 2,23 & 2,04 & 3,72 & 3,77 & 3,72 & 3,84 \\
\hline $\begin{array}{l}\text { Farelo de soja } 4 \\
\text { Soybean meal } 4\end{array}$ & 2,68 & 3,30 & 0,57 & 0,73 & 1,34 & 1,46 & 2,01 & 2,11 & 3,90 & 3,92 & 3,90 & 4,03 \\
\hline $\begin{array}{l}\text { Farelo de soja } 5 \\
\text { Soybean meal } 5\end{array}$ & 2,60 & 3,23 & 0,63 & 0,72 & 1,42 & 1,43 & 1,88 & 2,08 & 3,47 & 3,84 & 3,47 & 3,93 \\
\hline $\begin{array}{l}\text { Soja integral tostada } \\
\text { Toasted integral soybean }\end{array}$ & 3,15 & 2,18 & 0,44 & 0,51 & 1,29 & 1,03 & 1,66 & 1,52 & 3,65 & 2,58 & 3,65 & 2,50 \\
\hline $\begin{array}{l}\text { Soja micronizada } \\
\text { Micronized soybean }\end{array}$ & 2,41 & 2,71 & 0,53 & 0,62 & 1,03 & 1,23 & 1,67 & 1,80 & 2,39 & 3,22 & 2,39 & 3,39 \\
\hline Média (Mean) & 2,71 & 3,00 & 0,55 & 0,67 & 1,32 & 1,34 & 1,91 & 1,95 & 3,51 & 3,56 & 3,51 & 3,63 \\
\hline
\end{tabular}

${ }^{1}$ Equações propostas por Rodrigues et al (2002) (Equations proposed by Rodrigues et al., 2002): Lys = - 0,32161 + 0,06731PB $\left(R^{2}=0,99\right) ;$ Met = 0,02263 + 0,01323PB Met + cys $=0,07894+0,02563 P B\left(R^{2}=0,94\right) ; T h r=0,20930+0,03533 P B\left(R^{2}=0,95\right) ; A r g=-0,39589+0,08022 P B\left(R^{2}=0,95\right) A r g^{2}=-2,19880+0,11509 P B$ $+0,01702 \mathrm{EE}\left(R^{2}=0,98\right)$.

2 Conteúdo de aminoácidos essenciais totais obtidos por meio da análise dos alimentos.

3 Est - valores estimados pelas equações de predição.

2 Total indispensable amino acids content obtained by the feeds analysis.

${ }^{3}$ Est - Estimated values by the prediction equations. 
cada equação apresentada. As estimativas para arginina para a soja integral tostada e soja micronizada, por sua vez, foram aproximadamente $30 \%$ inferiores ou superiores, respectivamente, aos valores analisados nesta pesquisa, estando, portanto, fora da faixa recomendada pela literatura, que é de 10\% (Goldflus, 2001).

Quando se comparam os valores dos aminoácidos digestíveis estimados pelas equações e aqueles calculados por meio dos coeficientes de digestibilidade, obtidos no ensaio de metabolismo (Tabela 8), nota-se que o comportamento médio das variações entre os valores observados e estimados aumentou, até mesmo dos aminoácidos estimados de forma adequada em valores totais. Neste contexto, a arginina apresentou estimativas médias de 10,9 a 13,7\% em relação a cada equação testada e os aminoácidos totais, de 1,4 e 3,4\%. Este fato decorreu, possivelmente, da variação nos coeficientes de digestibilidade obtidos neste trabalho e aqueles encontrados por Rodrigues et al. (2002). Ressalta-se também que, em média, apenas a metionina + cistina e a treonina apresentaram estimativas dentro dos 10,0\% descritos por Goldflus (2001).

No entanto, se considerados os valores médios observados e aqueles estimados pelas equações apenas para as amostras de farelo de soja, nota-se que, à exceção da lisina e metionina, cujas estimativas médias foram superiores a $15,0 \%$, as equações para metionina + cistina, treonina e arginina digestível foram eficientes na estimativa dos valores, com percentuais 7,6\% inferior, 1,7 e 7,0\% superiores, respectivamente.

Ressalta-se que as equações propostas por Rodrigues et al (2002) foram desenvolvidas para um grupo de alimentos, o que, em alguns casos, pode comprometer os valores estimados para os alimentos individualmente, semelhantemente ao que ocorreu com as estimativas dos aminoácidos das amostras de soja integral tostada e soja micronizada nesta pesquisa. Neste contexto, o NRC (1998) ressalta que o ideal é estimar individualmente as equações de predição para alimentos, com altos coeficientes de determinação, para que possam estimar valores de aminoácidos aceitáveis.

De forma geral, as equações propostas no NRC (1998), para o farelo de soja (Tabela 9), foram menos eficientes em predizer os valores de aminoácidos que aquelas apresentadas por Rodrigues et al. (2002), uma vez que as diferenças entre os valores médios analisados e estimados foram de 22,4; 33,3; 11,3 e 5,0\%, respectivamente, para lisina, metionina, metionina + cistina e treonina. Portanto, somente a treonina esteve na faixa de $10 \%$ descrita por Goldflus (2001).

Essas diferenças podem, em parte, ser explicadas pelos baixos coeficientes de correlação entre as variáveis nas equações, que variaram de 65 a $81 \%$, de modo que a treonina apresentou valor mais elevado $(0,81)$, justificando sua melhor estimativa. Pode-se destacar também a diferença entre os farelos utilizados para estimar as equações propostas no NRC (1998) e aqueles usados neste trabalho, pois sabe-se que clima, tipo de solo, região geográfica e variedades influem de forma significativa no valor nutricional dos alimentos. Além disso, o fato de se trabalhar com equações específicas para determinado alimento não pode ser garantia de que estas estimem de maneira satisfatória os valores de aminoácidos deste alimento, quando cultivados em diferentes condições.

Tabela 8 - Estimativas do conteúdo de LYS, MET, MET + CYS, THR e ARG digestíveis da soja integral e subprodutos, por meio de equações de predição, em função da composição química dos alimentos (\% MS) ${ }^{1}$

Table 8 - Estimates of digestible LYS, MET, MET + CYS, THR e ARG contents of the full fat soybean and byproducts by the prediction equations based on the chemical composition (\%DM)

\begin{tabular}{|c|c|c|c|c|c|c|c|c|c|c|c|c|}
\hline \multirow{2}{*}{$\begin{array}{l}\text { Alimento } \\
\text { Feed }\end{array}$} & \multicolumn{2}{|c|}{ Lys } & \multicolumn{2}{|c|}{ Met } & \multicolumn{2}{|c|}{ Met + Cys } & \multicolumn{2}{|c|}{ Thr } & \multicolumn{2}{|c|}{ Arg } & \multicolumn{2}{|c|}{$\operatorname{Arg}^{2}$} \\
\hline & Obs. $^{2}$ & $\mathrm{Est}^{3}$. & Obs. & Est. & Obs. & Est. & Obs. & Est. & Obs. & Est. & Obs. & Est. \\
\hline Farelo de soja 1 (Soybean meal 1) & 2,46 & 2,99 & 0,53 & 0,67 & 1,44 & 1,24 & 1,80 & 1,84 & 3,03 & 3,67 & 3,03 & 3,75 \\
\hline Farelo de soja 2 (Soybean meal 2) & 2,61 & 3,03 & 0,52 & 0,67 & 1,16 & 1,26 & 1,61 & 1,86 & 3,48 & 3,72 & 3,48 & 3,79 \\
\hline Farelo de soja 3 (Soybean meal 3) & 2,51 & 2,99 & 0,52 & 0,67 & 1,28 & 1,24 & 1,96 & 1,84 & 3,33 & 3,67 & 3,33 & 3,76 \\
\hline Farelo de soja 4 (Soybean meal 4) & 2,52 & 3,10 & 0,55 & 0,69 & 1,31 & 1,28 & 1,75 & 1,90 & 3,62 & 3,81 & 3,62 & 3,96 \\
\hline Farelo de soja 5 (Soybean meal 5) & 2,39 & 3,04 & 0,61 & 0,68 & 1,34 & 1,26 & 1,69 & 1,87 & 3,14 & 3,74 & 3,14 & 3,86 \\
\hline Soja int. tostada (Toasted integral soybean) & 2,85 & 2,05 & 0,43 & 0,48 & 1,25 & 0,91 & 1,50 & 1,36 & 3,17 & 2,53 & 3,17 & 2,43 \\
\hline Soja micronizada (Micronized soybean) & 2,14 & 2,55 & 0,50 & 0,58 & 1,00 & 1,09 & 1,46 & 1,62 & 2,15 & 3,14 & 2,15 & 3,38 \\
\hline Média (Mean) & 2,50 & 2,82 & 0,52 & 0,63 & 1,25 & 1,18 & 1,68 & 1,76 & 3,13 & 3,47 & 3,13 & 3,56 \\
\hline
\end{tabular}

${ }^{1}$ Equações propostas por Rodrigues et al (2002) (Equations proposed by Rodrigues et al., 2002): Lys = - 0,29472 + 0,06319PB $\left(R^{2}=0,98\right) ;$ Met = 0,02884 + 0,01228PB $\left(R^{2}=0,93\right) ;$ Met + cys =0,08974 +0,02220PB $\left(R^{2}=0,91\right) ; \operatorname{Thr}=0,15813+0,03235 P B\left(R^{2}=0,95\right) ; A r g=-0,32129+0,07681 P B\left(R^{2}=0,96\right) ; A r g^{2}=-2,7699$ $+0,12416 \mathrm{~PB}+0,02312 \mathrm{EE}\left(\mathrm{R}^{2}=0,95\right)$.

2 Obs - conteúdo de aminoácidos digestíveis verdadeiros obtidos por meio dos coeficientes de digestibilidade determinados in vivo com galos adultos cecotomizados.

${ }^{3}$ Est - valores estimados pelas equações de predição.

${ }^{2}$ Obs - Digestible amino acids content of the full fat soybean and byproduct calculated by the digestibility coefficients determined "in vivo" with cecotomized cockerels.

${ }^{3}$ Est - Estimated values by the prediction equations. 
Tabela 9 - Estimativas dos conteúdos de lisina, metionina, metionina + cistina (MET + CIS) e treonina totais do farelo de soja, determinados por meio de equações de predição, em função da composição química dos alimentos propostas no NRC (1998) (\% MN) ${ }^{1}$

Table 9 - Estimates of total lysine, methionine, methionine plus cystine (Met+Cys) and threonine contents of soybean and byproducts by the prediction equations based on the chemical composition by NRC (1998) (as-fed basis)

\begin{tabular}{|c|c|c|c|c|c|c|c|c|}
\hline \multirow{2}{*}{$\begin{array}{l}\text { Alimento } \\
\text { Feed }\end{array}$} & \multicolumn{2}{|c|}{ Lys } & \multicolumn{2}{|c|}{ Met } & \multicolumn{2}{|c|}{ Met + Cys } & \multicolumn{2}{|c|}{ Thr } \\
\hline & Obs. $^{2}$ & Est. & Obs. & Est. & Obs. & Est. & Obs. & Est. \\
\hline Farelo de soja 1 (Soybean meal 1) & 2,29 & 2,91 & 0,48 & 0,67 & 1,34 & 1,37 & 1,79 & 1,85 \\
\hline Farelo de soja 2 (Soybean meal 2) & 2,60 & 2,97 & 0,50 & 0,69 & 1,12 & 1,39 & 1,70 & 1,89 \\
\hline Farelo de soja 3 (Soybean meal 3) & 2,41 & 2,90 & 0,51 & 0,67 & 1,24 & 1,37 & 1,99 & 1,85 \\
\hline Farelo de soja 4 (Soybean meal 4) & 2,40 & 3,02 & 0,51 & 0,70 & 1,20 & 1,41 & 1,80 & 1,92 \\
\hline Farelo de soja 5 (Soybean meal 5) & 2,34 & 2,98 & 0,57 & 0,69 & 1,28 & 1,40 & 1,69 & 1,89 \\
\hline Média (Mean) & 2,41 & 2,95 & 0,51 & 0,68 & 1,24 & 1,38 & 1,79 & 1,88 \\
\hline
\end{tabular}

${ }_{1}^{1}$ Lys $=-0,0810+0,0644 \mathrm{~PB}(r=0,78)$; Met $=0,0170+0,0141 \mathrm{~PB}(r=0,65) ;$ Met+ cys $=0,1470+0,0263 \mathrm{~PB}(r=0,60) ; \mathrm{Thr}=0,0810+0,0381 \mathrm{~PB}(r=0,81)$

${ }^{2}$ Obs - conteúdo de aminoácidos essenciais totais obtidos por meio da análise dos alimentos (Obs - total essential amino acids content obtained by feedstuffs analysis).

${ }^{3}$ Est - valores estimados pelas equações de predição (Est - estimated values by the prediction equations).

\section{Conclusões}

A digestibilidade verdadeira média dos aminoácidos essenciais e não-essenciais foi de 91,1 e 90,7\%, respectivamente. Com exceção das sojas integrais (tostada e micronizada), as equações de predição dos aminoácidos totais e digestíveis, propostas por Rodrigues et al. (2002), permitiram estimativas adequadas dos aminoácidos metionina + cistina, treonina e arginina. Por sua vez, à exceção da treonina, as equações propostas pelo NRC (1998) foram menos eficientes nas estimativas dos aminoácidos totais dos farelos de soja avaliados, quando comparados aos valores analisados nesta pesquisa.

\section{Literatura Citada}

ALBINO, L.T.F.; ROSTAGNO, H.S.; TAFURI, M.L. et al. Determinação dos valores de energia metabolizável aparente e verdadeira de alguns alimentos para aves, usando diferentes métodos. Revista da Sociedade Brasileira de Zootecnia, v.21, n.6, p.1047-1058, 1992.

ALBINO, L.F.T.; COELHO, M.G.R.; RUTZ, F. et al. Valores energéticos e de triptofano de alguns alimentos determinados, em aves jovens e adultas. Pesquisa Agropecuária Brasileira, v.22, n.11/12, p.1301-1306, 1987.

ARABA, M.; DALE, N.M. Evauation of protein solubility as an indicator of underprocessing of soybean meal. Poultry Science, v.69, n.10, p.1749-1752, 1990.

BELLAVER, C.; PARSONS, C.; EASTER, R.A. Estimativas da digestibilidade verdadeira de ingredientes de alimentos, obtida com galos cecotomizados em alimentação forçada. Pesquisa Agropecuária Brasileira, v.33, n.5, p.731-736, 1998.

BRASIL. MINISTÉRIO DA AGRICULTURA, DO ABASTECIMENTO E DA REFORMA AGRÁRIA - MAARA. Normas e padrões de nutrição e alimentação animal. Brasília: Secretaria de Desenvolvimento Rural - SDR; Departamento de Tecnologia e Produção Animal - DTPA, 2000. 152p.

DALE, N. Formulando com soya sobreprocesada. Indústria Avícola, v.44, n.3, p.52-53, 1997.

DEGUSSA, AG. Digestible amino acids in feedstuffs for poultry. Frankfurt: 1993. 18p.

FISCHER JR., A.A.; ALBINO, L.F.T.; ROSTAGNO, H.S. et al. Determinação dos coeficientes de digestibilidade e dos valores de aminoácidos digestíveis de diferentes alimentos para aves. Revista Brasileira de Zootecnia, v.27, n.2, p.307-313, 1998. GOLDFLUS, F. Ingredientes derivados do processamento da soja aplicados na nutrição animal. In: SIMPÓSIO SOBRE MANEJO E NUTRIÇÃO DE AVES E SUÍNOS, 2001, Campinas. Anais... Campinas: Colégio Brasileiro de Nutrição Animal, 2001. p.97-188.

NATIONAL RESEARCH COUNCIL - NRC. Nutrient requeriments of poultry. 9.ed. National Academy of Science, 1994. 155p.

NATIONAL RESEARCH COUNCIL - NRC. Nutrient requirements of swine. 3.ed. National Academy of Science, 1998. 189p.

PARSONS, C. M. Digestible amino acids for poultry and swine. Animal Feed Science and Technology, v.59, n.1-3, p.147153, 1996.

PARSONS, C.M.; FERNANDEZ, S.R.; YE ZANG. Dietary formulation with cottonseed meal on a total amino acid versus digestible amino acid basis. Poultry Science, v.74, n.7, p.1168-1179, 1995.

PUPA, J.M.R.; LEÃO, M.I.; CARVALHO, A.U. Cecectomia em galos sob anestesia local e incisão abdominal. Arquivo Brasileiro de Medicina Veterinária e Zootecnia, v.50, n.5, p.531-535, 1998.

RHÔNE POULENC ANIMAL NUTRITION. Rhodimet ${ }^{\mathrm{TM}}$ nutrition $^{\mathrm{N}}$ guide. 2.ed. Anthony Cedex: 1993. 55p.

RODRIGUES, P.B.; ROSTAGNO, H.S.; ALBINO, L.F.T. et al. Aminoácidos digestíveis verdadeiros da soja e subprodutos da soja, determinados com galos adultos cecectomizados. Revista Brasileira de Zootecnia, v.31, n.4, p.1771-1782, 2002.

ROSTAGNO, H.S.; ALBINO, L.F.T.; DONZELE, J.L. et al. Tabelas brasileiras para aves e suínos: composição de alimentos e exigências nutricionais. Viçosa, MG: Universidade Federal de Viçosa, 2000. 141p.

ROSTAGNO, H.S.; FEATHERSTON, W.R. Estudos de métodos para determinar disponibilidade de aminoácidos em pintos. Revista da Sociedade Brasileira de Zootecnia, v.6, n.1, p.64-76, 1977.

ROSTAGNO, H.S.; PUPA, J.M.R.; PACK, M. Diet formulation for broilers based on total versus digestible amino acids. Journal Applied Poultry Research, v.4, n.2, p.293-299, 1995.

SIBBALD, I.R. A bioassay for available amino acids and true metabolizable energy in feedstuffs. Poultry Science, v.58, n.3, p.668-673, 1979.

SILVA, D.J. Análise de alimentos (métodos químicos e biológicos). 2.ed. Viçosa, MG: Universidade Federal de Viçosa, 1990. 165p.

TANKSLEY JR., T.D.; KNABE, D.A. Recent advances in animal nutrition. London: Butterworths: 1984. p.75-94.

Recebido: 16/3/2006 Aprovado: $1 / 6 / 2007$ 